\title{
111In-Bevacizumab Imaging of Renal Cell Cancer and Evaluation of Neoadjuvant Treatment with the Vascular Endothelial Growth Factor Receptor Inhibitor Sorafenib
}

\author{
Ingrid M.E. Desar" ${ }^{* 1}$, Alexander B. Stillebroer ${ }^{* 2,3}$, Egbert Oosterwijk ${ }^{2}$, William P.J. Leenders ${ }^{4}$, Carla M.L. van Herpen ${ }^{1}$, \\ Winette T.A. van der Graaf ${ }^{1}$, Otto C. Boerman ${ }^{3}$, Peter F.A. Mulders², and Wim J.G. Oyen ${ }^{3}$ \\ ${ }^{I}$ Department of Medical Oncology, Radboud University Nijmegen Medical Centre, Nijmegen, The Netherlands; ${ }^{2}$ Department of \\ Urology, Radboud University Nijmegen Medical Centre, Nijmegen, The Netherlands; ${ }^{3}$ Department of Nuclear Medicine, Radboud \\ University Nijmegen Medical Centre, Nijmegen, The Netherlands; and ${ }^{4}$ Department of Pathology, Radboud University Nijmegen \\ Medical Centre, Nijmegen, The Netherlands
}

Clear cell renal cell cancer (ccRCC) prominently expresses vascular endothelial growth factor-A (VEGF-A), and new treatment strategies for renal cell cancer (RCC) aim at the inhibition of VEGF-VEGF receptor signaling. This study explores the ability of ${ }^{111} \mathrm{In}$-bevacizumab scintigraphy to depict RCC and to evaluate response to neoadjuvant treatment with sorafenib, a VEGF receptor inhibitor. Methods: The ability to depict RCC with ${ }^{111}$ In-bevacizumab scintigraphy was tested in 14 patients scheduled to undergo a tumor nephrectomy; of these, 9 RCC patients were treated in a neoadjuvant setting with sorafenib (400 mg orally twice a day). In the latter group, baseline and posttreatment ${ }^{111} \mathrm{In}$-bevacizumab scans were compared. The intratumoral distribution of ${ }^{111}$ In-bevacizumab was determined scintigraphically ex vivo in a 1-cm lamella of the resected tumorous kidney. Expression of VEGF-A, glucose transporter1 , carbonic anhydrase IX, $\alpha$-smooth-muscle actin, and Ki67 was determined by immunohistochemistry and compared with the local concentration of ${ }^{111}$ In-bevacizumab. Additionally, the VEGF-A content in tumor samples was determined quantitatively by enzyme-linked immunosorbent assay. Results: In all 5 non-neoadjuvant-treated patients, preferential accumulation of ${ }^{111} \mathrm{In}$-bevacizumab was observed in the tumors. All ccRCC lesions with enhanced ${ }^{111}$ In-bevacizumab targeting expressed high levels of VEGF-A. Treatment with sorafenib resulted in a significant decrease of ${ }^{111} \mathrm{In}$-bevacizumab uptake in the tumor in the patients with ccRCC (mean change, $-60.5 \%$; range, $+1.5 \%$ to $-90.1 \%)$. The decrease in uptake was due to destruction of the tumor neovasculature, whereas the VEGF-A expression remained intact. In the patient with papillary RCC, limited uptake without change after sorafenib was observed. Conclusion: RCC lesions were clearly delineated with ${ }^{111} \mathrm{In}$ bevacizumab scintigraphy. Neoadjuvant treatment with sorafenib resulted in a significant decrease of ${ }^{111}$ In-bevacizumab uptake in RCC. ${ }^{111}$ In-bevacizumab scintigraphy can be an attractive biomarker for response and needs further study.

Received Apr. 7, 2010; revision accepted Aug. 3, 2010.

For correspondence: Ingrid M.E. Desar, Department of Medical Oncology, Radboud University Nijmegen Medical Centre, P.O. Box 9101, 6500 HB Nijmegen, The Netherlands.

E-mail: i.desar@AlG.umcn.nl

${ }^{*}$ Contributed equally to this work.

COPYRIGHT ( 2010 by the Society of Nuclear Medicine, Inc.
Key Words: ${ }^{111} \mathrm{In}$-bevacizumab scintigraphy; renal cell carcinoma; sorafenib; angiogenesis inhibitor; biomarker

J Nucl Med 2010; 51:1707-1715

DOI: 10.2967/jnumed.110.078030

$\mathbf{T}$ reatment options for patients with metastatic renal cell cancer (RCC) have improved substantially in the past $5 \mathrm{y}$. Long mired in therapeutic nihilism because of chemotherapy resistance and modest effects of immunotherapy, metastatic RCC treatment has recently benefited from multiple active agents with marked clinical effects that have become available. Knowledge of underlying molecular characteristics identified the vascular endothelial growth factor-A (VEGF-A) and mammalian target of rapamycin pathways as fundamental to the biology of RCC. This biologic insight provided a rationale for targeting these growth factor signaling pathways in RCC. Small molecules inhibiting the tyrosine kinase portion of the intracellular receptor for VEGF have undergone extensive clinical testing. Two of these drugs, sunitinib and sorafenib (Nexavar; Bayer), are now widely used in clinical practice. These agents inhibit not only the VEGF receptor but also a broad spectrum of related receptor tyrosine kinases.

Because the development of angiogenesis inhibitors was not paralleled by appropriate evaluation methods for these kinds of drugs, new predictive biomarkers are urgently needed. Currently, CT scans are used mostly to diagnose RCC and evaluate treatment effects of tyrosine kinase inhibitors. CT scans are volumetric assessments of tumors and do not provide functional information. The Response Evaluation Criteria in Solid Tumors (RECIST), used to evaluate treatment response, are based on the sum of 1-dimensional measurements of the greatest diameter of the tumor or metastases $(1,2)$. However, treatment with tyrosine kinase inhibitors can result in necrosis and cavitation without a change in size, leading to an underestimation 
of therapeutic efficacy (3). Molecular imaging may be an alternative method to evaluate the efficacy of these new drugs. In clear cell RCC (ccRCC), a loss of function of the Von Hippel-Lindau gene product leads to accumulation of the hypoxia-inducible factor $1 \alpha$, with subsequent upregulation of hypoxia-inducible factor target genes, including VEGF-A (4-6). The accumulation and upregulation are independent from hypoxia and result in highly vascularized tumors. Therefore, molecular imaging of VEGF-A in ccRCC might be achieved and might provide functional information about the effects of tyrosine kinase inhibitor treatment (4-6).

Scintigraphy using bevacizumab (Avastin; Roche) may be an interesting approach to the imaging of VEGF-producing tumors. Bevacizumab is a humanized monoclonal antibody directed against all VEGF-A isoforms, inhibiting angiogenesis by preventing VEGF-A from binding to and activating its receptors. VEGF-A is the best-characterized member of the VEGF family and is considered the predominant and most critical regulator of neovascularization in various tumor types (7). Alternative splicing results in at least 6 isoforms of VEGF-A: $\mathrm{VEGF}_{121}, \mathrm{VEGF}_{145}, \mathrm{VEGF}_{165}$, $\mathrm{VEGF}_{183}, \mathrm{VEGF}_{189}$, and $\mathrm{VEGF}_{206}$. In tumors, $\mathrm{VEGF}_{121}$ and $\mathrm{VEGF}_{165}$, and to a lesser extent $\mathrm{VEGF}_{189}$, are predominant $(8,9)$. $\mathrm{VEGF}_{165}$ and $\mathrm{VEGF}_{189}$ are cell- or matrix-associated isoforms and are angiogenic, whereas $\mathrm{VEGF}_{121}$ is freely diffusible and mainly induces vasodilatation and vascular permeability $(8,10,11)$. Accumulation of antibodies such as bevacizumab in tumors is the result of multiple factors, such as antigen density, circulating antigen concentration, and tumor physiologic parameters such as microvessel density, vessel permeability, blood flow, and interstitial fluid pressure. In 2 animal studies and 1 clinical study, tumorspecific accumulation of radiolabeled bevacizumab has been described (12-14). Remarkably, no correlation between VEGF-A levels in plasma or tumor tissue extracts and bevacizumab uptake was observed in a study in patients with metastatic colorectal cancer (14). In that study, plasma VEGF-A was determined in a sample obtained just before labeled bevacizumab administration. The scintigraphy was performed 1 wk after tracer administration. Tumor VEGF-A was determined directly after surgery, at $10 \mathrm{~d}$ after tracer injection.

In the present study, the potential of ${ }^{111}$ In-bevacizumab to image VEGF-A-expressing ccRCC tumors was studied in 14 patients with primary RCC scheduled to undergo tumor nephrectomy. Nine of these patients were treated with sorafenib for $4 \mathrm{wk}$ in a neoadjuvant setting. The effect of sorafenib on ${ }^{111}$ In-bevacizumab uptake in RCC was explored in these patients.

\section{MATERIALS AND METHODS}

\section{Study Design}

Ten patients with suspected primary RCC and baseline enhanced ${ }^{111}$ In-bevacizumab uptake in the renal tumors were treated with sorafenib for $4 \mathrm{wk}$, after which ${ }^{111}$ In-bevacizumab scintigraphy was repeated, to observe the effect of sorafenib on ${ }^{111}$ In-bevacizumab uptake. As a control, ${ }^{111}$ In-bevacizumab scintigraphy was performed before surgery in 5 patients with suspected primary RCC and scheduled to undergo tumor nephrectomy (Fig. 1).

Exclusion criteria for all patients were prior use of bevacizumab or drugs targeting the VEGF or VEGF receptor, prior anticancer therapy, uncontrolled comorbidity, pregnancy, and lactation. For the sorafenib-treated group, adequate bone marrow (white blood cells $\geq 3.5 \times 10^{9} / \mathrm{L}$, platelets $\geq 100 \times 10^{9} / \mathrm{L}$, and hemoglobin $\geq$ $6 \mathrm{mmol} / \mathrm{L}$ ) and renal (serum creatinine $\leq 2 \times$ the upper limit of normal) and hepatic (total bilirubin $\leq 1.5 \times$ the upper limit of normal and aspartate aminotransferase and alanine aminotransferase $\leq 2.5 \times$ the upper limit of normal [ $\leq 5 \times$ in case of liver metastases]) function were required. The study was approved by the regional internal review board. Written informed consent was obtained from all patients.

\section{Study Drug}

Sorafenib (400 mg) was administered orally twice a day during 4 wk before nephrectomy. Dose interruptions and reductions were allowed when adverse events were grade 3 or higher according to the Common Toxicity Criteria (National Cancer Institute).

For scintigraphic imaging, bevacizumab was conjugated with isothiocyanatobenzyl diethylenetriaminepentaacetic acid (DTPA), as described previously (15). Kits containing $1 \mathrm{mg}$ of bevacizumab-DTPA conjugate in $1.0 \mathrm{~mL}$ of $0.15 \mathrm{M}$ citrate buffer, $\mathrm{pH} 5.5$, ready for radiolabeling were stored at $-20^{\circ} \mathrm{C}$ until use. The dose of $1 \mathrm{mg}$ was selected for imaging because optimal accumulation in VEGF-A-expressing tumors is obtained at relatively low antibody protein doses (13). On referral of a patient, a kit was labeled with $100 \mathrm{MBq}$ of ${ }^{111}$ In (Covidien). Radiochemical purity of all preparations used in this study exceeded $95 \%$, as determined by instant thin layer chromatography. The immunoreactivity was assessed in an enzyme-linked immunosorbent assay (ELISA), as described previously (16).

\section{Imaging}

In the control group, $10 \mathrm{~d}$ before nephrectomy, patients were intravenously injected with $1 \mathrm{mg}$ of DTPA-conjugated bevacizumab labeled with $100 \mathrm{MBq}$ of ${ }^{111}$ In within $1 \mathrm{~h}$ after preparation. A whole-body scan was acquired directly and at $7 \mathrm{~d}$ after injection, using a double-head $\gamma$-camera (E-Cam; Siemens Inc.), equipped with parallel-hole medium-energy collimators (symmetric 15\% window over 172 and $247 \mathrm{keV}$ ) and a scan speed of $8 \mathrm{~cm} / \mathrm{min}$
FIGURE 1. Treatment schedule for sorafenib-treated group.

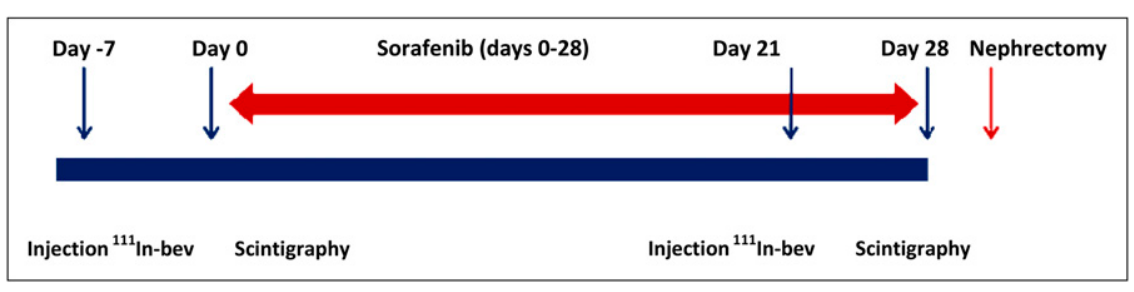


(day 0 ) and $4 \mathrm{~cm} / \mathrm{min}$ (day 7). This 7-d interval was based on our previous work in which we observed the best tumor-to-background ratios in the scans that were acquired at $7 \mathrm{~d}$ after injection $(17,18)$. To allow quantification of antibody targeting to the tumor, a known aliquot of the injected dose in an Adams phantom was scanned simultaneously. Targeting of the radiolabeled monoclonal antibody in tumor tissue was determined by comparing the accumulation of the radiolabeled antibody in the RCC tumors with that in the contralateral kidney. In addition, the targeting of ${ }^{111}$ In-bevacizumab was scored semiquantitatively, as described previously (19). In brief, regions of interest were drawn around tumors and the normal kidney on the planar images. ${ }^{111}$ In-bevacizumab targeting was expressed as the percentage injected dose per tissue weight (as measured on baseline CT), assuming a tissue density of $1.0 \mathrm{~g} / \mathrm{mL}$.

Patients underwent nephrectomy at $10 \mathrm{~d}$ after ${ }^{111}$ In-bevacizumab administration. After tumor nephrectomy, a 1-cm-thick slice containing both normal kidney tissue and tumor tissue was obtained from the surgical specimen; distribution of the radiolabeled antibody was then imaged for $45 \mathrm{~min}$ on the $\gamma$-camera, and the sample was cut into $1 \mathrm{~cm}^{3}$ slices. The radioactivity in each of the samples was quantified in a well-type $\gamma$-counter, and thereafter tissue blocks were processed for immunohistochemical analysis.

In the sorafenib-treated group, the same imaging procedures were used. ${ }^{111}$ In-bevacizumab was administered at $7 \mathrm{~d}$ before the start of sorafenib treatment and after $21 \mathrm{~d}$ of treatment, each time followed by a scintigraphy scan after $7 \mathrm{~d}$. The sorafenib treatment was discontinued on the day of the last scan. Tumor nephrectomy was performed at $3 \mathrm{~d}$ after the end of sorafenib treatment, and the tissue was processed in the same manner as for the controls. Only blood biodistribution was visualized on the early scans in the control group. Thus, in the sorafenib-treated group we chose to no longer perform ${ }^{111}$ In-bevacizumab scintigraphy directly after administration of the labeled bevacizumab because it provided no additional information but instead increased the burden to the patients.

\section{Immunohistochemical Analysis}

Vessel density and VEGF-A expression were determined immunohistochemically in $4-\mu \mathrm{m}$ formalin-fixed and paraffinembedded sections by staining with anti-CD31 (monoclonal antibody Jc70A; Dako) and anti-VEGF monoclonal antibody G153-694 (Pharmingen), respectively. An antibody against $\alpha$-smooth muscle actin ( $\alpha$-SMA) (Sigma) was used for the detection of pericytes as a measure of vessel maturation. To detect carbonic anhydrase IX (CAIX) expression, the samples were stained with the anti-CAIX antibody G250 (Wilex AG). As a marker of proliferation, Ki67 was stained (clone SP6; Lab Vision Corp.). Expression of VEGFA, CAIX, and CD31 were scored semiquantitatively on a scale ranging from undetectable $(-)$ to low $( \pm)$, moderate $(+)$, high $(++)$ and very high $(+++)$.

\section{VEGF-A Levels in Tumor Extracts}

In the control group, 10- $\mu \mathrm{m}$ cryosections of each sample of the kidney slice were pooled in radio immunoprecipitation assay buffer $(150 \mathrm{mM} \mathrm{NaCl} ; 10 \mathrm{mM}$ Tris-HCl, pH 7.4; $1 \mathrm{mM}$ ethylenediaminetetraacetic acid; $1 \%$ NP-40; and $0.5 \%$ sodium deoxycholate) containing a protease inhibitor cocktail (Roche) and heparin (5 U/ $\mathrm{mL}$ ) to release matrix-bound isoforms of VEGF-A. Sections were homogenized, incubated on ice for $20 \mathrm{~min}$, and centrifuged $(800 \mathrm{~g}$, $20 \mathrm{~min}, 4^{\circ} \mathrm{C}$ ) to prepare a clear lysate. The Bradford method
(Biorad) was used to determine protein concentration. To measure VEGF-A, a 4-antibody sandwich ELISA, which detects all VEGFA isoforms, was used (20). Data were expressed as nanograms of VEGF per milligram of protein for each sample, thus providing information about the VEGF-A levels in normal kidney tissue and in tumor samples.

\section{VEGF-A levels in Plasma}

In all patients, blood samples were collected to determine circulating VEGF-A concentrations before injection of ${ }^{111}$ In-bevacizumab. In the sorafenib-treated group, a blood sample was also taken after $21 \mathrm{~d}$ of treatment with sorafenib, before injection of the labeled bevacizumab. Plasma samples were stored at $-80^{\circ} \mathrm{C}$ within $1 \mathrm{~h}$ after venipuncture. Plasma concentrations were measured with the 4-antibody sandwich ELISA (20). This ELISA measures the unbound VEGF in plasma, which is relevant for the formation of immune complexes with radiolabeled bevacizumab.

\section{Statistics}

In the control group, based on the Kolmogorov-Smirnov and Shapiro-Wilk tests, we used the nonparametric 2-tailed Spearman $\rho$-test to analyze the correlation between VEGF-A values and ${ }^{111}$ In-bevacizumab uptake per sample and in each tumor. In the sorafenib-treated group, a nonparametric Wilcoxon signed-ranked test was performed to assess the change in ${ }^{111}$ In-bevacizumab uptake in the tumor before and after sorafenib treatment. A 2-tailed paired $t$ test on log-transformed values was used to determine the change in VEGF plasma levels. A $P$ value of less than 0.05 was considered significant.

\section{RESULTS}

\section{Patients}

In the control group, pathologic examination of the surgical specimens of all 5 patients (mean age, $61 \mathrm{y}$; 3 men, 2 women) revealed ccRCC. In 1 specimen, a sarcomatoid component was observed (patient 4); this patient also had pulmonary metastases at presentation. One patient had a synchronous renal oncocytoma (patient 5) (Table 1).

Ten patients were included, one of whom was unevaluable because the tumor proved to be not an ccRCC but an oncocytoma. Nine ccRCC patients were treated with sorafenib ( 2 women, 7 men; median age, 61 y; range, 4578 y; all with Eastern Cooperative Oncology Group scores of 0-1) after showing enhanced ${ }^{111}$ In-bevacizumab accumulation. Pathologic examination identified $8 \mathrm{ccRCC}$ and 1 papillary RCC (Table 1). Metastases, as assessed on a preoperative CT scan, were found in 6 patients. Neoadjuvant treatment with sorafenib was safe and well tolerated, although 3 patients needed a dose interruption and subsequent dose reduction (200 $\mathrm{mg}$ twice a day), because of grade 3 skin toxicity according to the Common Toxicity Criteria (3.0; National Cancer Institute). No additional toxicities were noted after nephrectomy.

\section{Scintigraphic Imaging of RCC with ${ }^{111}$ In-Bevacizumab}

Immediately after intravenous injection, ${ }^{111}$ In-bevacizumab distributed in the vascular system and in well-perfused 


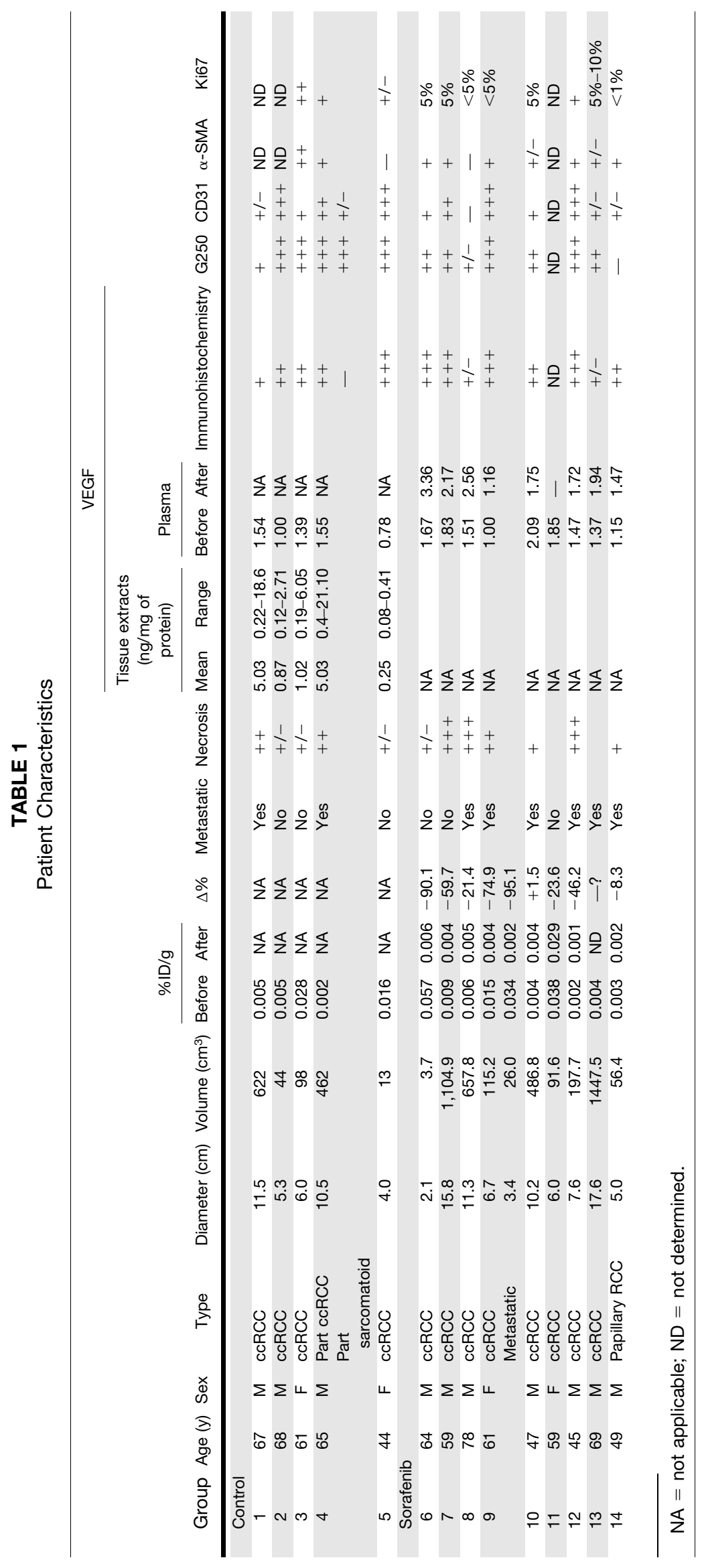


organs such as the liver and spleen, as judged by ${ }^{111} \mathrm{In}$ bevacizumab images. The tumors were not visualized on these early images of any of the 14 patients. In all 14 patients, preferential accumulation of ${ }^{111} \mathrm{In}$-bevacizumab was observed in the tumors at $7 \mathrm{~d}$ after the injection.

The mean ${ }^{111}$ In-bevacizumab uptake in ccRCC calculated from the images was $0.015 \%$ injected dose per gram $(\% \mathrm{ID} / \mathrm{g})$ of tumor tissue, as quantified by the images (median, $0.006 \% \mathrm{ID} / \mathrm{g}$; range, $0.002-0.028 \% \mathrm{ID} / \mathrm{g}$ ). In patient 4 , enhanced accumulation was also observed in the pulmonary metastases (Table 1). The scintigraphic images of this patient are shown in Figure 2A. Patient 5, who had a synchronous ccRCC and renal oncocytoma, showed only enhanced targeting of the ccRCC. The baseline uptake of ${ }^{111} \mathrm{In}$-bevacizumab in the papillary RCC was relatively low, as compared with the mean baseline uptake in the ccRCC tumors (0.003 vs. $0.019 \% \mathrm{ID} / \mathrm{g})$.

In all 5 controls, preferential accumulation of ${ }^{111} \mathrm{In}$ bevacizumab in the tumorous regions was observed, as established by ex vivo scintigraphy of the $1-\mathrm{cm}$ slice taken from the tumorous kidneys. Further quantification of ${ }^{111} \mathrm{In}$ bevacizumab uptake revealed that the antibody accumulated in areas of viable tumor tissue, whereas necrotic parts showed lower concentrations of the antibody (Fig. 2B). The quantitative values and measurements of the $\% \mathrm{ID} / \mathrm{g}$ of tumor tissue are summarized in Table 1.

After treatment with sorafenib, a significant reduction in ${ }^{111}$ In-bevacizumab accumulation in the tumors was observed in 7 patients with histologically proven ccRCC (mean, 0.019 $\% \mathrm{ID} / \mathrm{g}$ vs. $0.007 \% \mathrm{ID} / \mathrm{g}$; Table 1 ; Fig. 3). In 1 patient, only the pretreatment scan was available because of technical problems at the time of the second scan. ${ }^{111}$ In-bevacizumab targeting did not change in the patient with papillary RCC. In the ccRCC patients, a mean decrease of $60.5 \%$ for ${ }^{111} \mathrm{In}$ bevacizumab uptake was observed (median, $-46.2 \%$; range, $+1.5 \%$ to $-90.1 \% ; P=0.011$ ). The pattern of uptake of the

${ }^{111}$ In-labeled antibody in the tumor slices corresponded well with macroscopic discernable vital and necrotic areas in the tumor, with a relatively high uptake in the vital areas and low uptake in the necrotic areas (Fig. 4). The overall tumor uptake as derived from the quantitative analysis of the images is shown in Table 1. Tumors resected from sorafenib-treated patients contained much larger necrotic areas than tumors of similar size from untreated patients. Only 1 of 6 sorafenib-treated patients with metastases had pulmonary metastases larger than $1 \mathrm{~cm}$; this patient was, therefore, a candidate for ${ }^{111}$ In-bevacizumab uptake evaluation in the metastasis. In this patient, the uptake in the metastasis decreased from 0.034 to $0.002 \% \mathrm{ID} / \mathrm{g}$ of tumor tissue (reduction, $>95 \%$ ).

\section{Immunohistochemical Analysis}

The results of the immunohistochemical analyses of the viable parts of the tumors are presented in Table 1 and Figure 5.

Control Group. All ccRCCs with enhanced ${ }^{111}$ In-bevacizumab accumulation stained positively for VEGF-A. The oncocytoma of patient 5, in which no accumulation of ${ }^{111}$ In-bevacizumab was found, contained minimal amounts of VEGF-A. High expression of VEGF-A was found in viable parts of the tumors, whereas more necrotic parts and normal kidney tissue showed only limited VEGF-A expression. CAIX expression was high in all viable ccRCC areas. The RCC tumors of patients 2-5 highly expressed CD31 on their vasculature, although not in the sarcomatoid dedifferentiated part of the tumor of patient 4. Low CD31 expression, indicating low vessel density, was observed in the tumor of patient 1 .

Sorafenib-Treated Group. All treated tumors expressed VEGF-A. Notably, VEGF-A was also found in necrotic tumor parts. In general, the vessel density was low, as evidenced by low CD31 staining (Fig. 5), with a relatively large fraction of the remaining vessels lined with $\alpha$-SMApositive cells (mature vessels). Most of these vessels showed an abnormal aspect with apoptotic cells. All ccRCCs were CAIX-positive; the papillary RCC was CAIX-negative. Cell proliferation, as judged by Ki67 staining, was low (Table 1).

\section{VEGF Analysis}

Besides the VEGF staining, we also measured the concentration of VEGF-A in samples of the kidney slices from nontreated patients. The concentration of VEGF-A in
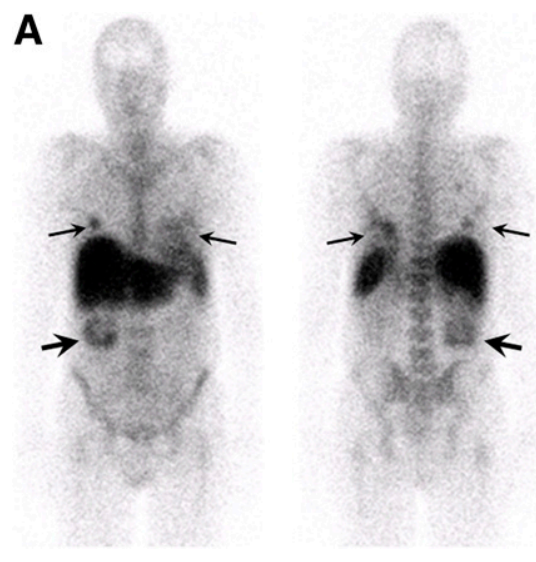

B

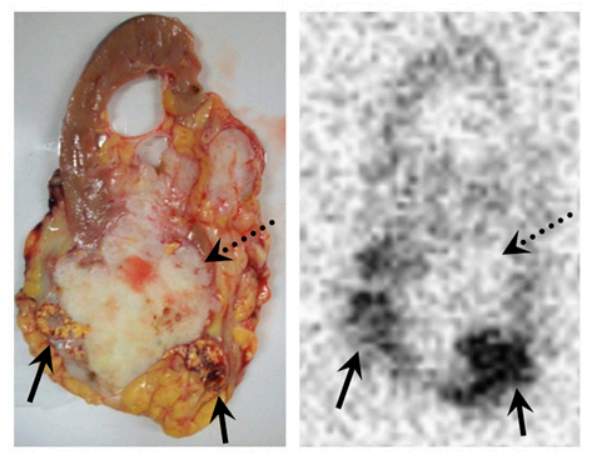

FIGURE 2. Scintiscans of VEGF-A expression in ccRCC lesions with ${ }^{111}$ In-bevacizumab (patient 4). (A) Anterior and posterior wholebody images showing CCRCC in right kidney (large arrow) and pulmonary metastases (small arrows). (B) Photograph and scintiscan of resection specimen showing enhanced VEGF-A targeting of ${ }^{111}$ In-bevacizumab in cCRCC part of renal tumor (solid arrows) and decreased uptake in sarcomatoid part (dashed arrows), corresponding with immunohistochemistry results showing low vessel density in sarcomatoid part, which hampered ${ }^{111} \mathrm{In}$ bevacizumab delivery. 
FIGURE 3. Anterior and posterior $111 \mathrm{ln}$ bevacizumab scintiscans at baseline $(A)$ and after 4 wk of treatment with sorafenib (B). Decrease of ${ }^{111}$ In-bevacizumab uptake, more enhanced in central parts of tumor, is shown (arrows).
A

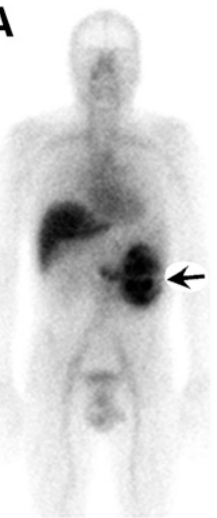

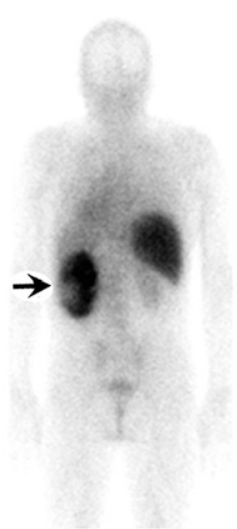

B

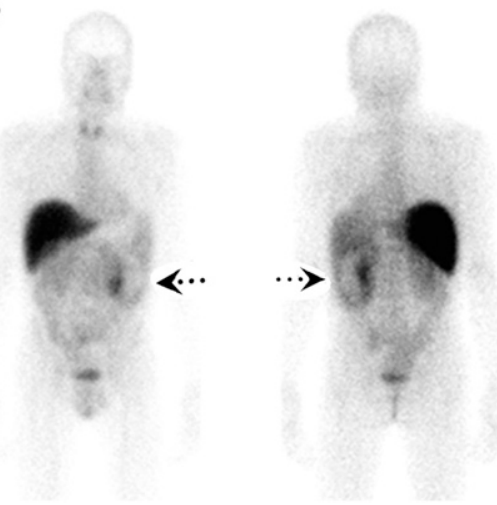

these specimens ranged from 0.08 to $21.10 \mathrm{ng} / \mathrm{mg}$ of protein (mean, $3.67 \mathrm{ng} / \mathrm{mg}$ of protein). In extracts containing at least $50 \%$ vital RCC, as estimated on the sections of each tumor sample stained with hematoxylin and eosin, the mean VEGFA concentration was $3.24 \mathrm{ng} / \mathrm{mg}$ of protein $(n=40$; range, $0.12-13.24 \mathrm{ng} / \mathrm{mg}$ of protein), and in those extracts containing at least $75 \%$ vital tumor, the mean VEGF-A concentration was $3.12 \mathrm{ng} / \mathrm{mg}$ of protein $(n=20$; range, $0.24-7.27 \mathrm{ng} / \mathrm{mg}$ of protein). Interestingly, in extracts containing a maximum of $20 \%$ vital tumor, and thus with a high fraction of necrotic material $(>80 \%)$, the mean VEGF-A concentration was higher: $5.11 \mathrm{ng} / \mathrm{mg}$ of protein $(n=47$; range, 0.12 $21.10 \mathrm{ng} / \mathrm{mg}$ of protein). Surprisingly, a negative correlation between the VEGF-A concentration in the tissue extract and the ${ }^{111}$ In-bevacizumab uptake in the tissue cubicles was found $(n=89$; Spearman $\rho, r=-0.274 ; P=0.010)$. This negative correlation was even more significant in a subgroup
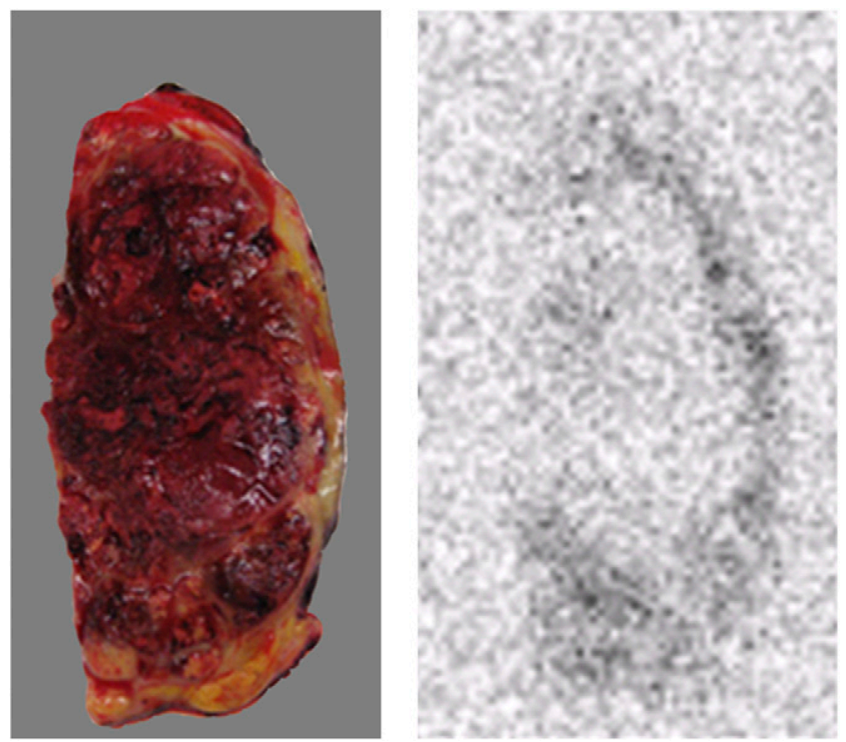

FIGURE 4. Photograph and scintiscan of surgical specimen of patient 7 , showing extensive necrosis in almost whole tumor, except for more vital borders, at which VEGF-A targeting of ${ }^{111} \mathrm{In}$-bevacizumab is preserved. analysis when only extracts containing at least $75 \%$ viable tumor were analyzed $(n=20 ; r,-0.565 ; P=0.009)$. In the samples containing a maximum of $20 \%$ viable tumor, no correlation between VEGF concentration and ${ }^{111}$ In-bevacizumab uptake was found $(n=47 ; r,-0.231 ; P=0.119)$. Accumulation of ${ }^{111}$ In-bevacizumab in within tumors was heterogeneous and did not correlate with VEGF-A concentration of the same samples $(r=0.185, P=0.095)$. In

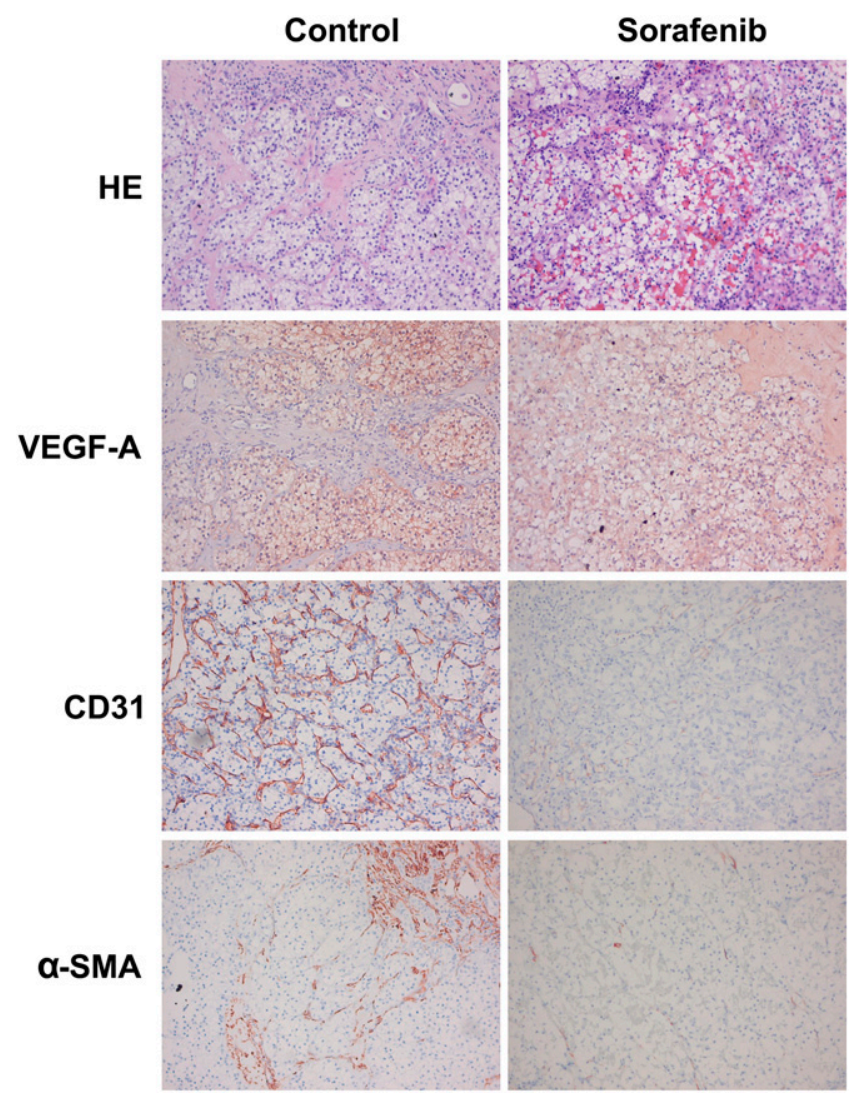

FIGURE 5. Immunohistochemical analyses. Neoadjuvant treatment with sorafenib resulted in enhanced necrosis (HE) and decreased vessel density (CD31), with excessive loss of young immature vasculature ( $\alpha$-SMA). No changes in VEGF-A expression were observed. $\mathrm{HE}=$ hematoxylin and eosin. 
patients 1 and 4, a negative correlation was found, whereas patients 2 and 5 showed a positive correlation between VEGF-A concentration and ${ }^{111}$ In-bevacizumab concentration or accumulation.

The mean VEGF plasma level in the control patients was $1.49 \mathrm{ng} / \mathrm{mL}$ (range, 0.78-2.71 ng/mL). A significant increase in VEGF plasma levels was found after $4 \mathrm{wk}$ of treatment with sorafenib (mean, $1.55 \mathrm{ng} / \mathrm{mL}$ vs. $2.02 \mathrm{ng} / \mathrm{mL}, P=$ 0.026). There was no correlation between tumor and plasma VEGF-A levels.

\section{DISCUSSION}

In the present study, we show that ${ }^{111}$ In-bevacizumab preferentially accumulated in primary RCC and its metastases and that treatment with sorafenib resulted in a dramatic decrease in ${ }^{111}$ In-bevacizumab accumulation, suggesting that sorafenib-induced (vascular) changes are reflected in the reduced accumulation of the radiolabeled antibody.

The reduced uptake of ${ }^{111}$ In-bevacizumab was not the consequence of reduced VEGF-A expression, because VEGF-A expression in the RCC lesions was comparable (irrespective of treatment) as evidenced by the immunohistochemical analyses. It is likely that the destruction of the tumor vasculature resulted in decreased ${ }^{111}$ In-bevacizumab delivery and consequently decreased accumulation. We observed extensive necrosis in the tumors after treatment with sorafenib-necrosis that was more extensive than that observed in untreated RCC tumors of similar size. The macroscopic pattern of viable and necrotic tissue regions corresponded with the intratumoral distribution of ${ }^{111} \mathrm{In}$ bevacizumab. Immunohistochemical analysis corroborated that the tumor vasculature was greatly altered after sorafenib treatment: a minimal presence of immature vessels was observed, as judged by CD31 or $\alpha$-SMA staining, and the remaining mature vessels were lined with apoptotic cells. In most tumors, the necrosis was in the central part of the tumors, whereas the more vital tumor areas were at the tumor periphery. Uptake of ${ }^{111}$ In-bevacizumab in these vital parts of the tumor was not enhanced. There was a 3-d interval between the ending of sorafenib treatment and tumor nephrectomy. Therefore, the observed immunohistochemical changes might not be a true reflection of treatment-induced features during sorafenib. However, this interval is short, and major changes within this interval are unlikely.

Vascular changes due to treatment with sorafenib are most likely quite dynamic. A time-dependent change in tumor vascularization during treatment with antiangiogenic drugs, starting with normalization of the vasculature and ending with loss of vasculature and necrosis, has been described previously (21). Tumor perfusion would increase temporarily with concomitant enhancement of ${ }^{111}$ In-bevacizumab uptake. However, at later stages, when necrosis prevails, perfusion is decreased and ${ }^{111}$ In-bevacizumab uptake will be reduced. Our results strongly suggest that after $4 \mathrm{wk}$ of sorafenib treatment the latter scenario prevails, which was also the case with ${ }^{125}$ I-cG250 accumulation in an RCC mouse model treated with several tyrosine kinase inhibitors, including sorafenib (22). Multiple tyrosine kinase inhibitors can be given in 2 approaches: as an antiangiogenic drug and as a signal transducer inhibitor, focused on optimization of the tumor microenvironment through sustained tumor vasculature normalization and reduced hypoxia to enhance combination therapy with, for example, chemotherapy or radiotherapy (23). The present study indicates that further investigations focusing on dosing and timing of (combined) treatment and imaging are warranted.

It is interesting to speculate on the observed vascular changes resulting from sorafenib treatment. Sorafenib treatment caused fewer effects on the more mature and larger tumor vessels. One could hypothesize that these remnant vessels play an important role in acquiring resistance against sorafenib and other angiogenesis inhibitors. Furthermore, the fact that more mature vessels were less affected by sorafenib might support sorafenib treatment at an earlier stage of disease, when more immature vessels are present - a possibility that is currently under investigation in large adjuvant trials such as the SORCE trial (24).

We found a negative correlation between VEGF-A expression and ${ }^{111}$ In-bevacizumab concentration in tissue. For ${ }^{111}$ In-bevacizumab VEGF-A expression, circulating VEGF-A levels, bevacizumab concentrations, and tumor delivery of bevacizumab are important parameters that also affect the extent of tumor accumulation. The relatively low VEGF-A concentrations in samples with at least $75 \%$ vital tumor, combined with the high ${ }^{111}$ In-bevacizumab uptake in these samples, suggest that antigen expression is not the major parameter determining bevacizumab accumulation. Other factors, such as tumor vessel characteristics, appear to be more important. In earlier studies, we have shown that high antigen expression is a prerequisite for high antibody uptake but that fulfillment of other conditions is equally pivotal to establishing high antibody uptake (25). The intratumoral heterogeneity, intrapatient differences, and lack of bevacizumab uptake in a benign VEGF-expressing oncocytoma support this notion. Vessel characteristics can influence ${ }^{111}$ In-bevacizumab uptake in 2 ways: nonspecific accumulation of antibodies due to enhanced vascular permeability or insufficient delivery of antibodies due to poor vascularization. Studies in nude mice with human tumors did not demonstrate any accumulation when an antigenblocking dose of antibody was coadministered, suggesting that accumulation of bevacizumab in the tumor was antigen-specific $(12,13)$. Obviously, it is difficult to extrapolate results obtained in xenografted mice to patients with RCC.

Two other explanations for the negative correlation between VEGF-A levels and uptake of ${ }^{111}$ In-bevacizumab can be considered. First, one could hypothesize that the relatively low bevacizumab dose used in this study (1 mg) 
could be saturated by circulating VEGF-A $(1.49 \mathrm{ng} / \mathrm{mL})$. However, this low antibody protein dose is still more than a 100-fold molar excess, as compared with the VEGF concentration in plasma. This molar excess remains after sorafenib-induced increases of plasma VEGF-A (mean at baseline, $1.55 \mathrm{ng} / \mathrm{mL}$, vs. mean after sorafenib, $2.02 \mathrm{ng} / \mathrm{mL}$ ). Only $1 \%$ of the administered bevacizumab activity could be complexed with plasma VEGF-A. Therefore, we did not examine immune complex formation. Second, RCC is known to preferentially express $\mathrm{VEGF}_{189}, \mathrm{VEGF}_{165}$, and $\operatorname{VEGF}_{121}(26,27)$. Bevacizumab binds all VEGF isoforms, but tumor accumulation of ${ }^{111} \mathrm{In}$-bevacizumab is mediated by the cell- and matrix-bound VEGF-A isoforms, such as $\operatorname{VEGF}_{165}$ and $\operatorname{VEGF}_{189}(10,11)$. In contrast, the ELISA used to determine VEGF content in the tumor tissue detects all isoforms of VEGF-A, because no isoform-specific ELISA was available. Therefore, the freely diffusible $\mathrm{VEGF}_{121}$ can be a disturbing factor. If vital RCCs produce relatively high levels of $\mathrm{VEGF}_{121}$, while the $\mathrm{VEGF}_{165}$ and $\mathrm{VEGF}_{189}$ amounts are similar, then the negative correlation would be explained, because washout of immune complexes of bevacizumab and $\mathrm{VEGF}_{121}$ will occur. In the same way, when tumors have lower amounts of VEGF but relatively high levels of $\mathrm{VEGF}_{165}$ and $\mathrm{VEGF}_{189}$, ${ }^{111}$ In-bevacizumab uptake can be enhanced. The expression patterns of VEGF isoforms within RCCs do differ, and a relationship with tumor histology, tumor growth, or tumor stage has been suggested (26-29).

The implementation of therapeutics targeting the VEGF pathway, with their effects on tumor physiology, and the need for patient stratification, warrant imaging techniques that are able to determine VEGF expression in vivo and that provide functional information. ${ }^{111}$ In-bevacizumab scintigraphy could provide functional information about the effect of neoadjuvant treatment with sorafenib in RCC. The next step will be the comparison of ${ }^{111}$ In-bevacizumab scintigraphy not only to conventional $\mathrm{CT}$ scans defining response with the RECIST criteria but also to new approaches, such as incorporation of (modified) Choi criteria in RCC evaluations (30) or dynamic contrast-enhanced MRI (31).

\section{CONCLUSION}

${ }^{111}$ In-bevacizumab scintigraphy is able to depict RCC lesions. Furthermore, neoadjuvant treatment with sorafenib significantly reduces accumulation of ${ }^{111}$ In-bevacizumab in RCC lesions. ${ }^{111}$ In-bevacizumab is an attractive biomarker for clinical response and needs further study.

\section{ACKNOWLEDGMENTS}

We thank Gerben Franssen, Lieke Joosten, Mirjam de Weijert, and Anneke Geurts-Moespot for their help on the VEGF tumor extracts and Cathy Maass and Gursah Kats for their help on the immunohistochemical stainings. This study was supported by grant KUN 2007-3940 from the
Dutch Cancer Society and an educational grant by Bayer. The trial registration number is NCT00602862.

\section{REFERENCES}

1. Therasse P, Arbuck SG, Eisenhauer EA, et al. New guidelines to evaluate the response to treatment in solid tumors. European Organization for Research and Treatment of Cancer, National Cancer Institute of the United States, National Cancer Institute of Canada. J Natl Cancer Inst. 2000;92:205-216.

2. Eisenhauer EA, Therasse P, Bogaerts J, et al. New response evaluation criteria in solid tumours: revised RECIST guideline (version 1.1). Eur J Cancer. 2009;45:228-247.

3. Desar IM, van Herpen CM, van Laarhoven HW, Barentsz JO, Oyen WJ, van der Graaf WT. Beyond RECIST: molecular and functional imaging techniques for evaluation of response to targeted therapy. Cancer Treat Rev. 2009;35:309-321.

4. Takahashi A, Sasaki H, Kim SJ, et al. Markedly increased amounts of messenger RNAs for vascular endothelial growth factor and placenta growth factor in renal cell carcinoma associated with angiogenesis. Cancer Res. 1994;54:4233-4237.

5. Turner KJ, Moore JW, Jones A, et al. Expression of hypoxia-inducible factors in human renal cancer: relationship to angiogenesis and to the von Hippel-Lindau gene mutation. Cancer Res. 2002;62:2957-2961.

6. Raica M, Cimpean AM, Anghel A. Immunohistochemical expression of vascular endothelial growth factor (VEGF) does not correlate with microvessel density in renal cell carcinoma. Neoplasma. 2007;54:278-284.

7. Ferrara N, Carver-Moore K, Chen H, et al. Heterozygous embryonic lethality induced by targeted inactivation of the VEGF gene. Nature. 1996;380:439-442.

8. Kusters B, de Waal RM, Wesseling P, et al. Differential effects of vascular endothelial growth factor A isoforms in a mouse brain metastasis model of human melanoma. Cancer Res. 2003;63:5408-5413.

9. Roodink I, van der LJ, Kusters B, et al. Development of the tumor vascular bed in response to hypoxia-induced VEGF-A differs from that in tumors with constitutive VEGF-A expression. Int J Cancer. 2006;119:2054-2062.

10. Hoeben A, Landuyt B, Highley MS, Wildiers H, van Oosterom AT, de Bruijn EA. Vascular endothelial growth factor and angiogenesis. Pharmacol Rev. 2004;56:549-580.

11. Park JE, Keller GA, Ferrara N. The vascular endothelial growth factor (VEGF) isoforms: differential deposition into the subepithelial extracellular matrix and bioactivity of extracellular matrix-bound VEGF. Mol Biol Cell. 1993;4:13171326 .

12. Nagengast WB, de Vries EG, Hospers GA, et al. In vivo VEGF imaging with radiolabeled bevacizumab in a human ovarian tumor xenograft. $J$ Nucl Med. 2007;48:1313-1319.

13. Stollman TH, Scheer MG, Leenders WP, et al. Specific imaging of VEGF-A expression with radiolabeled anti-VEGF monoclonal antibody. Int $J$ Cancer. 2008;122:2310-2314.

14. Scheer MG, Stollman TH, Boerman OC, et al. Imaging liver metastases of colorectal cancer patients with radiolabelled bevacizumab: lack of correlation with VEGF-A expression. Eur J Cancer. 2008;44:1835-1840.

15. Brouwers AH, van Eerd JE, Frielink C, et al. Optimization of radioimmunotherapy of renal cell carcinoma: labeling of monoclonal antibody cG250 with ${ }^{131} \mathrm{I}$, ${ }^{90} \mathrm{Y},{ }^{177} \mathrm{Lu}$, or 186Re. J Nucl Med. 2004;45:327-337.

16. Collingridge DR, Carroll VA, Glaser M, et al. The development of $\left[{ }^{124} \mathrm{I}\right]$ iodinated-VG76e: a novel tracer for imaging vascular endothelial growth factor in vivo using positron emission tomography. Cancer Res. 2002;62:5912-5919.

17. Brouwers AH, Buijs WC, Oosterwijk E, et al. Targeting of metastatic renal cell carcinoma with the chimeric monoclonal antibody G250 labeled with ${ }^{131} \mathrm{I}$ or ${ }^{111}$ In: an intrapatient comparison. Clin Cancer Res. 2003;9:3953S-3960S.

18. Scheer MGW, Stollman TH, Boerman OC, et al. Radiolabeled bevacizumab as a tracer of VEGF-expression in patients with colorectal liver metastasis. Eur J Cancer, in press.

19. Visser E, Postema E, Boerman O, Visschers J, Oyen W, Corstens F. Software package for integrated data processing for internal dose assessment in nuclear medicine (SPRIND). Eur J Nucl Med Mol Imaging. 2007;34:413-421.

20. Span PN, Grebenchtchikov N, Geurts-Moespot J, Westphal JR, Lucassen AM, Sweep CG. EORTC Receptor and Biomarker Study Group Report: a sandwich enzyme-linked immunosorbent assay for vascular endothelial growth factor in blood and tumor tissue extracts. Int J Biol Markers. 2000;15:184-191.

21. Jain RK. Normalization of tumor vasculature: an emerging concept in antiangiogenic therapy. Science. 2005;307:58-62.

22. Oosterwijk-Wakka JC, Kats-Ugurlu G, Leenders WP, et al. Effect of tyrosine kinase inhibitor treatment of renal cell carcinoma on the accumulation of carbonic anhydrase IX-specific chimeric monoclonal antibody cG250. BJU Int. 2010 . 
23. Maity A, Bernhard EJ. Modulating tumor vasculature through signaling inhibition to improve cytotoxic therapy. Cancer Res. 2010;70:2141-2145.

24. Kapoor A, Gharajeh A, Sheikh A, Pinthus J. Adjuvant and neoadjuvant smallmolecule targeted therapy in high-risk renal cell carcinoma. Curr Oncol. 2009;16 (suppl 1):S60-S66.

25. Steffens MG, Oosterwijk E, Zegwaart-Hagemeier NE, et al. Immunohistochemical analysis of intratumoral heterogeneity of $\left[{ }^{131} \mathrm{I}\right] \mathrm{cG} 250$ antibody uptake in primary renal cell carcinomas. Br J Cancer. 1998;78:1208-1213.

26. Nicol D, Hii SI, Walsh M, et al. Vascular endothelial growth factor expression is increased in renal cell carcinoma. J Urol. 1997;157:1482-1486.

27. Tomisawa M, Tokunaga T, Oshika Y, et al. Expression pattern of vascular endothelial growth factor isoform is closely correlated with tumour stage and vascularisation in renal cell carcinoma. Eur J Cancer. 1999;35:133-137.
28. Jacobsen J, Grankvist K, Rasmuson T, Ljungberg B. Different isoform patterns for vascular endothelial growth factor between clear cell and papillary renal cell carcinoma. BJU Int. 2006;97:1102-1108.

29. Rivet J, Mourah S, Murata H, et al. VEGF and VEGFR-1 are coexpressed by epithelial and stromal cells of renal cell carcinoma. Cancer. 2008;112:433442.

30. Smith AD, Lieber ML, Shah SN. Assessing tumor response and detecting recurrence in metastatic renal cell carcinoma on targeted therapy: importance of size and attenuation on contrast-enhanced CT. AJR. 2010;194:157165.

31. Hahn OM, Yang C, Medved M, et al. Dynamic contrast-enhanced magnetic resonance imaging pharmacodynamic biomarker study of sorafenib in metastatic renal carcinoma. J Clin Oncol. 2008;26:4572-4578. 